\title{
Article \\ Robot-Enhanced Language Learning for Children in Norwegian Day-Care Centers
}

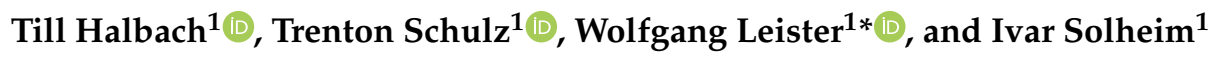 \\ 1 Norsk Regnesentral (Norwegian Computing Centre), Oslo, Norway; \\ \{Till.Halbach,Trenton.Schulz,Wolfgang.Leister\}@nr.no \\ * Correspondence: Wolfgang.Leister@nr.no
}

\begin{abstract}
We transformed the existing learning program Language Shower, which is used in some Norwegian day-care centers in the Grorud district of Oslo municipality, into a digital solution using an app for smartphone or tablet with the option for further enhancement of presentation by a NAO robot. The solution was tested in several iterations and multiple day-care centers over several weeks. Measurements of the children's progress across learning sessions indicate a positive impact of the program using a robot as compared to the program without robot. In-situ observations and interviews with day care center staff confirmed the solution's many advantages, but also revealed some important areas for improvement. In particular, the speech recognition needs to be more flexible and robust, and special measures have to be in place to handle children speaking simultaneously.
\end{abstract}

Keywords: Social robot; mobile app; e-learning; language education; kindergarden, pre-school

\section{Introduction}

In the Grorud district of the city of Oslo, up to $80 \%$ of the children in the day-care centers come from a minority or have a migration background. These children often speak a different language at home. This issue is exasperated when the children begin in school as they are behind their peers when it comes to Norwegian vocabulary. In spite of measures to help improve children's language learning in the Grorud district's pre-schools, about $40 \%$ of the children starting in school in 2017 needed additional Norwegian language support [1]. New methods were needed.

One area to improve was building Norwegian vocabulary. The pedagogic staff in the Grorud district of Oslo developed the Language Shower specifically to build the children's vocabulary [1]. The idea of the program is to "shower" the children with Norwegian words every day over a longer period, with the goal to strengthen their oral and vocabulary skills. Currently, the program lasts one year, primarily targeting children aged four to six. The program also has a secondary target of the children's parents, who in many cases are in need of language training, too. Overall, Language Shower is designed as a supplement to other language learning measures in the district's day-care centers.

The municipality was interested in the possibility to use a social robot to play the role of the session leader in Language Shower sessions to assist the staff and increase the children's engagement. Social robots have recently become popular in a variety of applications ranging from entertainment over customer expedition to teaching and education [2]. We created a prototype for a robot-lead Language Shower to examine how social robots can enhance and support children's learning of a second language in three day-care centers in Grorud district. The development and trials were carried out between Autumn 2018 and Spring 2021, and the overall objective of the work was to enhance the Language Shower learning program in the district with a social robot.

Parts of the study reported in this paper are based on a pilot study by Fuglerud and Solheim [3]. They used a NAO robot [4] in two studies with children with autism spectrum 
disorder and with children that were second language learners, observing increased participation and involvement in language learning. Fuglerud and Solheim recommended to investigate the role of the robot in the overall pedagogical approach and its ethical implications in further studies. Further, the themes of engagement and participation, combination of tools, learning outcomes, the need for better speech recognition, language issues, session length and group size, personalisation and easy to use interface, and technical support are revisited in the current article.

We documented the initial work around this study earlier [5]; here we go into detail around implementing the digital Language Shower, the issues we faced, the actual study, and results. The remaining article is straight-forward. After the outlining background and related work (Section 1), we describe the components and content for the digital Language Shower (Section 2). Then, we describe how the trials were conducted (Section 3) and discuss the trials' results (Section 4). Finally, we conclude the article with possibilities for future improvement (Section 6).

\section{Background}

A social robot has been defined as an "... autonomous or semi-autonomous robot that interacts and communicates with humans by following the behavioral norms expected by the people with whom the robot is intended to interact" [6, p. 592]. Social robots can be used in a variety of ways in an educational setting for both adults [7] and school children [8]. Examples for children as the target learning group include using social robots as tools for enabling remote attendance [9], for children's groupwork to increase participation and proactiveness [10], or tele-teaching, where students can use an avatar to remotely attend classes and be present in the classroom [11]. Another application of social robots can be as tutors for stimulating scientific curiosity [12] or improving a child's engagement, motivation, and performance in subjects such as learning sign language [13]. Let us examine how social robots have been used for language education and how our work relates to the previous work. Then, we will examine some of the issues that one needs to consider when designing a solution that uses a social robot. Afterwards, we will discuss the Language Shower concept.

\subsection{Social robots in language instruction}

An early example of a social robot in language instruction was a humanoid robot deployed in an elementary school in Japan where children could interact with a robot during a break after lunch $[14,15]$. To help reduce the complexity of the interactions, the children were told that the robot could only speak English. This had the effect that it motivated the children to learn and use English, which lead to the children learning new English vocabulary.

In many of the language learning projects social robots have been used for learning English, German, Dutch, Italian, Spanish, Japanese, Korean and Persian [16,17]. There are not many studies addressing the use of social robots for learning Norwegian, which is a language used by fewer people, other than the work we build upon $[3,5]$.

Different strategies have been applied for different studies. For example, one study examined how a robot (a NAO) could help German children aged 4 to 7 to scaffold their second-language learning of English [18]. That is, a robot would provide additional explanations (a scaffold) about why choices are being made to help reinforce learning. The robot would also detect flagging motivation (via Wizard of Oz [19], that is, a person observing through NAO) and attempt to re-engage the child. Not all children completed the training, but the study showed that the robot could often re-engage children, and that the explanations were helpful to the group of children that completed the training. This study shows that robots can be useful for re-engaging training with children to keep motivation, and help in learning the language.

A recent survey [20] examined a number of studies that were done in Robot-Assisted Language Learning (RALL). The survey found that robots were often autonomous, teleoperated, or a combination of both. The robots served many different functions such as: being 
a conversation partner, an aid in building vocabulary, grammar, pronunciation, reading comprehension, writing clarity, or assessing language ability. Often the robot helped with vocabulary building (especially for a second language) or as a conversation partner. The robots had different forms: human-like, animal-like, or machine-like, and they often took the role as an assistant to the teacher or a peer or tutor. Often the learners in the studies had increased motivation and interest in language. But the survey found limitations: the robots were normally limited to a few sessions, the context was mostly children in the classroom in Asia, and the statistical power of the studies was low. Our digital Language Shower follows a similar set up building vocabulary as an assistant, and it adds an additional context of Norwegian day-care centers (a younger group than what was examined in the survey).

Another review [21] examined social robots for language learning, including studies focusing on word learning in preschool and young school-aged children. The review included 33 studies, thirteen of them focusing on word learning, and considered them related to learning outcome. Regarding learning outcome, the review concluded that the learning gains were similar between social robots and human teachers. The learning gain seemed to be generally low in most of the studies since, like the survey [20] above, the research design of many studies only included one session. The review also considered effects, such as effects on the learners' motivation, novelty effect, and social behavior. There appeared to be positive effects of social robots on learning-related emotions, but the novelty effect was often not considered in the reviewed studies, while other studies that did run longer reported a declining interest as time progressed.

From the review and survey, it appears that at least some results on learning-related emotions and learning gains might stem from the initial excitement (or novelty) of using the robot. Regarding social behavior, the existing evidence with respect to social robots' seems to be mixed, as it could increase children's engagement in learning tasks, as well as distracting them from learning. Still, there are a variety of issues to consider when using a social robot for language learning.

\subsection{Issues and challenges in using social robot for language learning}

One issue to consider when using social robots is the role the robot should have in the instruction. Determining and assigning roles in human-robot interaction (HRI) has often been a complex and dynamic process [22]. One study reported that children seemed to learn better when a NAO robot played the role of a peer instead of a teacher [23]. In other studies, robots acted as teaching assistants in the classroom. For example, RoboSapiens assisted teachers for five weeks in an elementary school when they read stories using different voices, lead recitals, provided friendly feedback, gave quizzes, and moved when students asked [24]. Similarly, a NAO robot was used with some children to read out vocabulary words with pictures shown on slides behind it, and pantomime the meanings, while also providing entertainment such as singing and dancing [8]. The results of the study showed that students learned faster and learned more words, compared to a control group.

Once a role is settled on, another issue can be how to structure the interaction. The structure can consist of several elements. Should a session be a group or a one-on-one interaction? One-on-one interactions provide better opportunities for tailoring the experience to the child, but there are also benefits to learning in a group environment. One also needs to determine optimal length of sessions, the number of words that should be trained per session, and the optimal age group for using robots. It is also important to consider how learning outcomes can be measured before, during, and after the robot session.

Another issue to consider is how the robot should behave socially during the instruction. A robot that behaves more seriously than playfully may fit better for serious tasks [25]. Although social behaviors can be effective for engaging students, they can distract children [26]. Furthermore, positive effects have been observed from personalizing 
the behavior of two autonomous robots that acted as learning companions for children [27].

One challenge mentioned often in studies and reviews was automatic speech recognition (ASR) for children $[3,16,17,20]$. Word recognition of children's speech in current solutions was two to five times worse than adults' speech [20]. Others have examined issues surrounding child speech recognition in human-robot interaction context [28]. The study examined how microphones and noise from the robot (a earlier version of NAO) (and the surrounding area) affected the recognition. Overall, better microphones raised the rate of recognition significantly, but it required that the audio be normalized before recognition, something that happened on the robot's built-in microphone automatically. The additional noise of the robot's servos did not seem to affect the recognition rate. Ultimately, the study noted that some larger issues exist including that most speech recognition engines are trained on and expect an adult voice instead of a child's voice, and that there is, in general, a much larger corpus of adult speech available for training. Although there have been improvements in the technology since these articles, we encountered issues as we developed the digital Language Shower as well.

Aware of some of the issues raised in the previous research, the L2TOR project [16] had a goal of creating a second language tutor using a social robot for children ages 5 and up. The project documented several different aspects that should be considered when developing such a solution. These aspects include pedagogical issues such as what words to teach; what ages to target; and how many words to teach. Other aspects were in childrobot interaction such as how to introduce the robot, the interaction context, the robots non-verbal and verbal behaviors, how to give feedback, and the importance of customizing the lessons to each child.

Then, the L2TOR project applied these guidelines and ran a study that examined how a NAO robot and tablet compared against only a tablet for teaching English words to Dutchspeaking children [29]. The tablet was used as a medium for the robot to communicate with the children to avoid issues with ASR. 194 Dutch children between the ages of 5 and 7 were split into groups that had seven lessons one-on-one with either a robot and tablet, a tablet only, or no technology. The robot group was further divided into groups where the robot used gestures to pantomime the words and one that did not. The study found that children could retain the words they had learned from the robot at the same level they could with a tablet and that there was no difference between groups where the robot used gestures or did not.

\subsection{The Language Shower concept}

The original Language Shower [30] was developed by the pedagogic staff in the Grorud district of the city of Oslo and is based on research from children's language development in school and pre-school. It contains of a set of Norwegian words that the children are supposed to know when they start in school. The entirety of words is split into topics, such as the human body, my family, home, and clothing. A typical week with Language Shower consists of one daily session lasting 15 minutes with seven to eight children and one or two staff. The program follows a basic two-week schedule; during the first week (five days), roughly ten new words are introduced and learned, and during the second week, these words are frequently repeated and used in various tasks. This pattern is then repeated with different words and topics.

Each word is accompanied by a picture, such that for instance "hand" comes with a picture of a human hand as visual aid. The staff controls which pictures are shown, explain words and tasks, and evaluate answers and each child's performance. The program also provided different tasks related to the words, like multiple-choice questions, mind mapping with synonyms and antonyms and other related terms, comparisons, clapping, and rhymes. The original version of Language Shower started with pictures presented on sheets of paper, but there was also an implementation in Powerpoint where the pictures were shown on a wall by means of a projector connected to a tablet PC. 


\section{The Digital Language Shower solution}

We transformed the existing Language Shower to a new digital format with a tablet app and an optional robot. We tried to follow the original learning program as closely as possible (with certain modifications to exploit the possibilities that the novel combination of app and robot gives). There were several reasons for this: (1) The original program has been revised multiple times to incorporate experiences made during daily use in day-care centers. (2) Keeping similarities between the old and the new format would make it easier to identify those factors that are potential causes for differences in learning. (3) Finally, we then could reuse a number of pictures and tasks from the existing Language Shower, giving a more efficient content development.

The entire project started with a small-scale pre-project in Autumn 2018 to assess the maturity of the technology [3]. Early in 2019, we conducted interviews and focus groups with (ordinary) staff from the day-care centers, the district's pedagogues and experts for language training, as well as parents, 14 parents in total, both with and without an immigrant background. The notes from the conversations were then structured and analyzed with regard to user requirements and functional requirements. The suggestions included to make provision for a dialog between the children and robot, and to include game elements to increase the children's motivation. The interviews also reinforced the need to involve the children's parents in the language teaching as the parents are crucial for maintaining the children's motivation at home. We observed through the interviews that many of the parents needed to learn or improve their own Norwegian skills.

The Digital Language Shower consists of an app for smartphones or tablet and a social robot. Both are interconnected. The software development was carried out in two major iterations: A beta version of the app and the robot were tested in a limited pilot in early 2020. The suggestions for enhancements from the trials were implemented along with bug fixes and new features before the main trial in Autumn 2020 and complemented with all necessary content. This resulted in the final version.

Previously, we have given a short outline of the solution and its components in a late-breaking report [5]. We will now present a detailed explanation of the content, the app, and the robot, starting with the Digital Language Shower requirements.

\subsection{Technical and functional requirements}

Finding the appropriate set of requirements was an involved process since there were multiple target groups: (1) the children in the day-care centers, (2) their parents and families at home, and (3) the pedagogical staff. In addition, there were security and privacy aspects to take into account, as well as the existing pedagogical framework, as detailed further below.

It was viewed early on as beneficial to combine the functionality of the app with the robot for several reasons. First, there needed to be an additional means to show the visual cues of the words the children were supposed to learn. Second, the children's families would not have a robot at home, but the availability of a smartphone is common in Norway. Third, learning can continue using the app regardless if the robot is connected or not (especially, in cases of a poor network connection). This also neatly addresses the principle of progressive enhancement, where the app provides the basic learning experience that is further enhanced by the robot if it is available.

Next, there were requirements from the day-care center staff. In practice, unexpected situations can easily arise, such as children talking simultaneously, touching the robot, and children who require extra attention, leading to the requirement of a non-autonomous robot. And, last but not least, the app could take over both the tasks of a content server and monitoring of the learning progression, as explained in Section 2. This removed the need for external dedicated servers and thus reduced the solution's complexity, costs, and maintenance efforts quite a bit.

An easy-to-use and cost-effective app appeared to solve all aforementioned demands. 


\subsection{Content}

This section explains the details of the content, including learning program and pedagogical concepts. By content, we refer to media files, such as images, sound effects, music, speech, choreographed robot movements, other robot instructions, and descriptions of how these media files are organized, interconnected, used, and presented. Content is both a representation and a description of digital assets for learning and, therefore, fills the app and the robot with "life".

\subsubsection{Course structure and modalities}

The Digital Language Shower program was designed as a course which is subdivided into course modules, also referred to as topics. The topics are, in turn, compound of lectures. A lecture consists of learning units. The semantic description of modules, topics, lectures, and learning units is stored in one file in JSON format. The content file contains the instructions for both app and robot, and it points to where the media files for images and audio are stored in the file system. Each semantic element holds a unique ID. The description also specifies the achievable score for each learning unit.

A lecture's maximum score is the sum of scores for its learning units, and a module's maximum score is accordingly the sum of scores of the module's lectures. It is further possible to specify a minimum-score requirement for entering a module or lecture, combined with particular content IDs; otherwise access to the lecture is locked until the score is achieved. This mechanism controls the order for accessing content and links this to the user's learning progress.

All learning units have in common that the user actively must choose to continue, e.g., by pushing a button. However, depending on the specification of learning unit, this choice may not be available without an answer, or before, say, a playback button has been pushed.

A learning unit may contain descriptions of alternative content called modalities, which are the most basic logical structures. The learning goal of a set of associated modalities is identical, but the learning means or measures typically differ. In this work, we have developed two modalities for the content: (1) app-only and (2) the app in combination with the robot The first modality describes the learning means for situations without robot, such as at home, or when robot and app are not interconnected while the second modality enhances the lectures with the robot, i.e., targeting day-care centers.

\subsubsection{Learning unit types}

We developed three basic types of learning units: (1) a plain narrative (listen or watch), (2) a multiple-choice question, and (3) a word collection. Figure 1 shows how these are displayed in the app. A basic characteristic of the learning units is whether they provide for one-way communication of knowledge or two-way interaction.

Plain narrative units (Figure 1(a)) imply no interaction. In the app-only modality, a plain narrative unit typically means the display of an image and a piece of text, or the playback of an audio file, containing for instance the pronunciation of a particular word. Using the app in conjunction with the robot, the same image is shown in the app, accompanied by the robot telling a story or playing a song and dancing. Regardless of the modality, the key element is the image, which is supposed to trigger the children's associative abilities.

A multiple-choice unit (Figure 1(b)) depends on interaction, that is, somebody answering the question. The app displays one or several images as a visual clue and, in the app modality, a textual question with two or multiple answering options as buttons. In the modality of the app and robot, the app shows only the image, and the robot takes over the dialog, i.e., poses the question and awaits the answer. Here, it is up to the content producer to specify how many times a question can be answered. In our study, we chose three trials for all oral multiple-choice questions. As an answer is given, the robot replies with what it has understood first, and whether the answer was correct. Thereafter, as long as it still accepts more answers: "Again". Should the answer still be wrong, the robot says 
the correct answer and comes with a couple of comforting words. A correct answer is awarded by the robot with a victory position, a little dance, clapping, or another funny move. In the app-only modality, it is only possible to answer once.

A word collection unit (Figure 1(c)) also relies on user interaction. The app displays a set of images, one at a time, typically with words to be be repeatedly trained. In the app-only modality, there also is a set of buttons, one for each word, and the user has to press the right button of the currently raised image. In the app and robot modality, the robot asks for the word of the current image, and evaluates the answer. The images shift after each answer, and the entire unit is finished after the last images has been presented. A word collection unit differs from the other units in that each wrong answer leads to a reduction in score, but the score cannot go below zero.

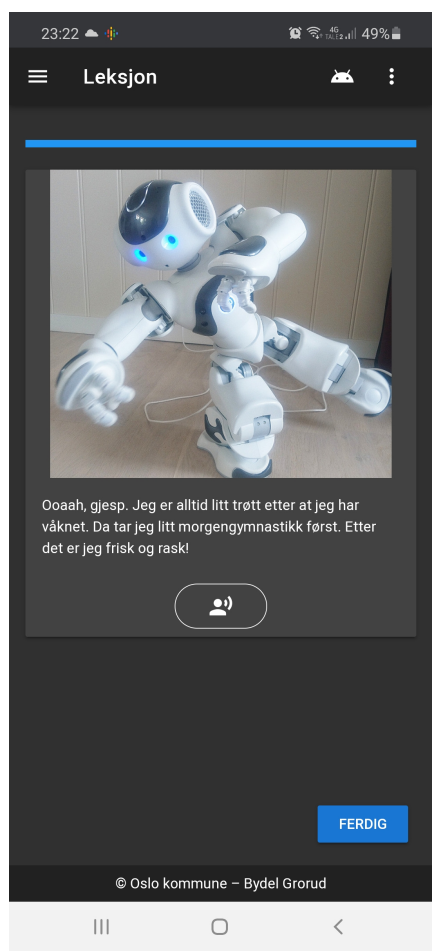

(a) Plain narrative

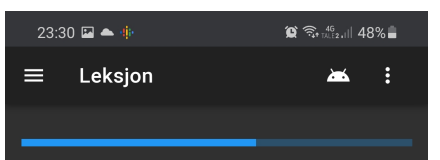

Dette er skikkelig vanskelig, så nå trenger jeg litt hjelp av deg! Av dyrene på bildet, hvilket dyr har munn?

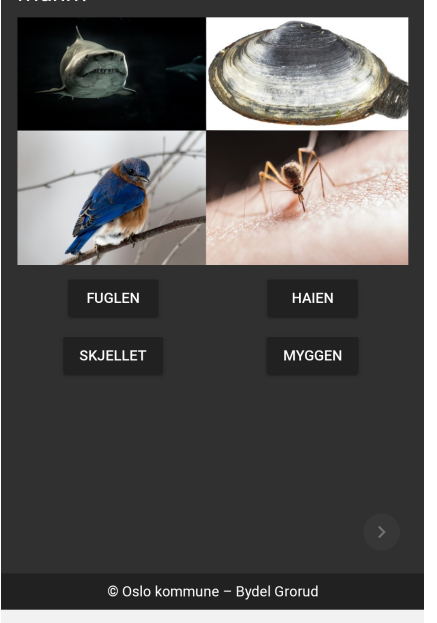

(b) Multiple-choice question

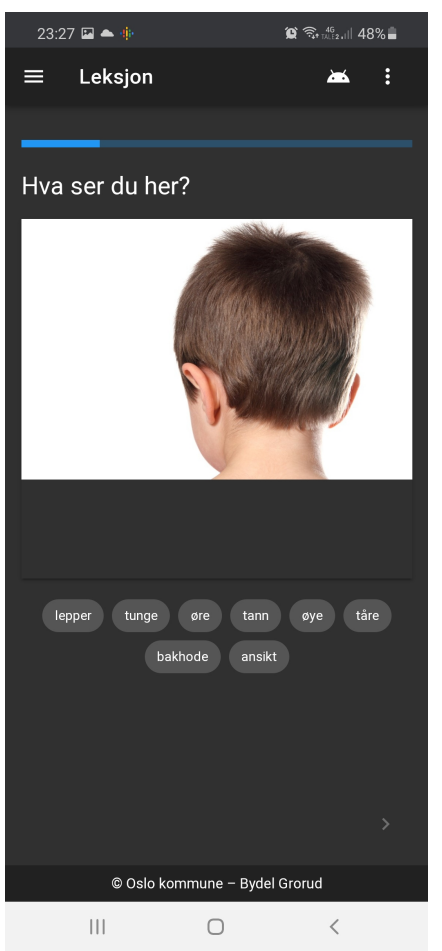

(c) Word collection

Figure 1. Screenshots of tasks / learning units in the app

\subsection{Pedagogical aspects}

We produced content for a six-week learning program in total; two weeks with the topic Clothes, and four weeks with My body. In terms of word count, this implied respectively 10, 11, and 12 new words in the word introduction weeks, each of which was followed by a repetition week.

We further extended Language Shower with a couple of new elements: There is a module called The robot with three lectures. In the first lecture, the robot says hello, and this lecture can be played the very first time the children meet the robot or when a session is started. In the second lecture, the robot pretends to just have woken up, plays back the opening song and dances. The opening song is supposed to be done before each Language Shower with the purposes to put the children in the right state of mind. The last lecture contains an ending sequence where the robot waves and says goodbye. This one is supposed to be played at the end of each language learning session, for the children to more easily accept that the session is over.

When there were not enough tasks in the original Language Shower, we added new content (five to eight tasks), with the goal to fill approximately 15 minutes for each session. 
We tried to vary the tasks as much as possible within the framework given by the original Language Shower program. In addition, the robot's moves, lights, audio, and phrases were randomly changed to achieve more variation. For example, the sentence the robot says to comfort students when an answer is incorrect has over six alternatives, which should minimize the effect of the robot repeating itself every single time. Finally, we also added one dance every other week, including appropriate moves and light effects. The music was purchased from professional producers.

A day where a new word was introduced started with a repetition of words learned earlier in the week (except on the first day of each week). Then, two to three new words were introduced, each new word had a combination of a multiple-choice question ("what do you see here") followed by a task to make a mind map for this new word. The mind map task is implemented as a plain narrative unit and solved by the group while the robot waits. The mind map activity relies heavily on the staff's guidance. Typically, the remaining tasks consist of pronouncing the words' first letter, clapping syllables, and finding rhymes. This is followed by associative tasks regarding color ("red shirt"), count ("three fingers"), size ("large legs"), position ("bottom left"), comparison to related phrases ("yellow like cheese"), and so on.

A repetition day starts with a word collection containing all words introduced during the week before. After this task, the focus turns to two to three selected words from the collection. A typical activity here is to go through the mind map for each word again and expand the map. Following this task, more tasks with associative and interactive activities are performed. The tasks here are typically more difficult than during the introduction week.

\subsection{Robot instructions}

The content file also contains robot instructions, either a single instruction or a sequence of instructions, such as (1) raise left arm, (2) play back a sound, then (3) say "correct". As a movement can happen concurrently with audio and speech, almost any robot behavior can be described by an instruction set like this. During the execution of a learning unit, the app splits a series of instructions and sends the robot single instruction messages. See Section 2.7 for a description of these.

An annotated example of a lecture description is given in Listing 1 with a plain narrative unit that consists of two modalities. The example shows Norwegian texts only, but in general each piece of text must be specified in all available languages. As speech is always in Norwegian, no further language specification is currently needed.

\subsection{Mobile app}

The municipality required that the app ran on Android and iOS operating systems. So, it was decided to implement the app as a progressive web application (PWA). Thus, the app can run on many smartphones, tablet, and PCs with a variety of screen sizes and orientations. PWAs are also great for rapid development and deployment.

The app consists of three main views (not counting Settings and About views): List of course modules (topics), module/list of lectures, and lecture/learning units. As for now, there are the three topics: Meet the teacher, Clothes, and Your body. A single lecture corresponds to one Language Shower session; i.e., the children are supposed to go through one lecture each day, or-in turn - the learning units contained in a lecture. An example for a lecture is: "New words: Underpants, boxer", and "Repetition: All previous words, particularly sock, tights". During a lecture, the user navigates from learning unit to learning unit by pressing the Next button in the screen's lower right corner.

The connection parameters to the robot can be personalized in the settings of the NAO. When the robot's chest button is pressed, it speaks its IP address, which, in turn, can be entered in the respective field in the app's settings. This is followed by an internal handshake between the app and the robot. If the handshake is successful, the robot icon in the app's lecture view will turn green. 
Listing 1: An example description of a lecture

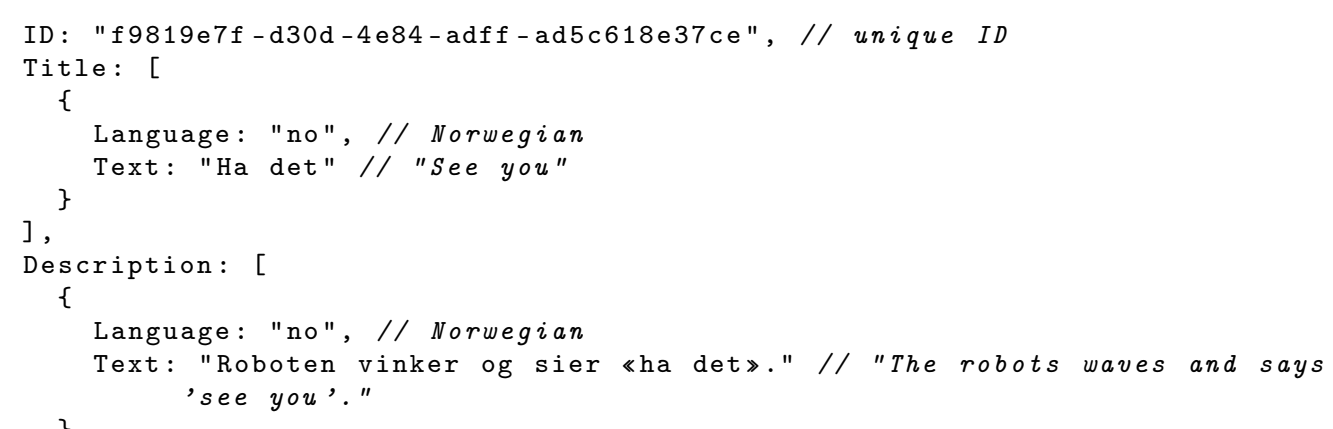
],

Illustration: "robot-waving.jpg", // image illustrating the lecture Requirements: [], // here, no requirements to enter lecture Units: [ // list with learning units contained in this lecture \{

ID : "d58e46ff-88ec-46d2-ae41-bf0ebe5584d3", // learning unit ID Score: 0, // maximum score Modalities: [

$\{$ // description for combination of robot and app Type: "narrative: robot + still", Still: "robot-talking.jpg", // image shown by the app Instructions: [ // for the robot \{

Type: "monologue", // robot doesn't wait for answers \} , 
The app also provides some gamification elements in the user interface. The lecture and course module scores are shown as the user has finished a lecture, and also later in the lecture listings and module listing. Some graphical user interface elements slide in and out of view in an animated, dynamic manner and are accompanied with sound effects. Correct answers produce a bell sound, and incorrect answers produce a buzzer sound.

\subsection{Monitoring progress}

After a learning unit is finished, important key parameters that measure the user's progress are collected and stored as a progress report inside the app's internal database in xAPI format by the Advanced Distributed Learning Initiative [31]. These measurements include starting time, unique user ID, unique learning unit ID, ending time, score, and maximum score. Hence, each progress report corresponds to a single learning unit and summarizes who has done which learning unit, when, for how long, and how successful the progress was. The app uses the database to keep track of which lectures a user already has done, and the scores for lectures, modules, and courses so far. As scores already achieved elsewhere can be retrieved, it is possible to control which lectures, modules, and courses should be available to the user based on earlier progress.

In the trials discussed in Section 3, the progress report was also sent to an external database called Learning Record Store (LRS), which was hosted on our servers so that we could track the progress remotely. As a side note, these progress reports allow for the possibility to track users' learning progress and to analyze the content for potential weaknesses. This has not been part of the current project.

\subsection{Robot}

The project used two NAO 6 robots that were semi-autonomous. That is, the robot would receive instructions from the app, perform its actions autonomously, and then wait for the next instructions. When building our version of the Language Shower, we had to determine how to best use the robots' speech capabilities and how to control the robot through the app.

\subsubsection{Speech recognition and speech pronunciation}

From the factory, NAO has the capabilities to speak and understand English, but one can install a Norwegian voice and speech recognition software. Given the voice that it uses, we assumed that the voice technology was based on Acapela's system. NAO's Norwegian pronunciation is not prefect, but it has improved from previous versions and is considered usable. Fortunately, it is possible to adjust pronunciation by adjusting the text to be pronounced. We tried Acapela's tags for controlling NAO's speech [32], but they did not seem to work. Eventually, we adjusted the regular text, including using accent marks, splitting words, and adding punctuation in creative ways to get an acceptable result. For example, the robot pronounced the location name "Grorud" as [groru'-], but it should actually be pronounced [gro:'ru-]. We adjusted the input text to be "Grô Rud", which is close enough. In other situations, we could not get the robot to pronounce the 's' sound correctly, and had to adjust the word accordingly. We recognize that this technique is fragile and could break on updates to the speech synthesis software [33]. However, this was the best we could do with the current set up and limited time for development.

As mentioned in Section 1.2, we ran into limitations with NAO's built-in automatic speech recognition (ASR), which was lacking sufficient documentation for Norwegian. NAO's Norwegian speech recognition could not understand words that are not in its dictionary, such as names. This limitation ruled out some simple dialogues. For example, the following construction would not be possible (emphasis added for word not in the dictionary):

Child: "Hello, Robot. My name is Suleiman."

Robot: "Hello Suleiman." 
This dialog would not work because the name Suleiman was not in the dictionary. It may have been possible to add these names, but there was no documentation for this.

Further, the ASR had difficulties distinguishing between simple words. For example, the Norwegian en (one) was misinterpreted as fem (five); occasionally even clearing one's throat was misinterpreted as "five." We considered lowering the confidence threshold for the recognition, but this even worsened the quality of the recognition.

We addressed this challenge by working with an expert from our project partner Innocom who implemented a solution using Google's Speech to Text service. This introduced a small delay, as speech was sent to Google's service for processing before the recognized text was returned. Our tests showed that the delay was negligible in most situations. There were also issues with Google's service, which we will detail later.

\subsubsection{Controlling the robot and the Language Shower}

To address the requirement for progressive improvement of the learning experience, it was decided that a pedagogue would control the Language Shower session through the tablet app. That is, the robot imitated the session leader, but it presented the instructions sent from the tablet app controlled by the pedagogue who actually lead the activities. Due to this dependency between the tablet app and speech recognition, the robot always needed to be connected to the network. In our study, we used an internal network at the day-care centers for the robot and the app.

The robot was connected to the app using the WebSocket protocol [34]. After the robot had received its IP-address from the router, and this address was added to the settings dialog in the app (Section 2.5), the app would send the robot one instruction at a time. The robot would then send a confirmation that it had completed the instruction before the next instruction was sent. The instructions were formalized in JSON and the communication served as an API between the robot and the app.

Listing 2: An instruction from the tablet app to the robot.

\{

"Title": "NR Tutor: App", // Message from the app

"Version": "0.9",

"AppID ": "...",

"DateTime": "2018-11-13T20:20:39+00:00",

"DeviceID": "...",

"Request": "Directive", // Type of instruction

"ObjectID": "6db6..611e", // Instruction UUID

"AnswerRequired": false, // No interaction required.

"Speech": "Hello everyone, I am Robbie", // Words to say.

"Gesture": "winner", // The gesture to perform.

"Light": "blink", // Effect for the eyes.

\},

"Color": "\\#ff0000" // The color the eyes should have.

Listing 2 shows a message from the app to the robot, and Listing 3 shows the corresponding response from the robot to the app. The most important fields in Listing 2 are: (1) Speech (Line 10): what the robot will say, (2) Gesture (Line 11): how the robot should move, (3) Light (Line 12): Control the lighting effect in the robot's eyes, and (4) Color (Line 13): Control the color of the robot's eyes.

The NAO's actions were programmed using Choregraphe [35]. Using the program, we created a content package, installed it on all the NAO's, and configured the package to start automatically. The package contained the code for the robot's actions and the WebSocket client. The robot does not perform any actions until it receives instruction from the app. If no instructions are received after ten minutes, the robot will go into it's resting (crouch) position to save battery and reduce wear on the servos. One could also change the robot into this position by touching the back of the robot's head.

There were some additional challenges with the specific version of NAO we used. NAO runs its own custom version of Linux (based on Gentoo), which could cause issues 
Listing 3: Confirmation response from the robot to the app's instruction in Listing 2.

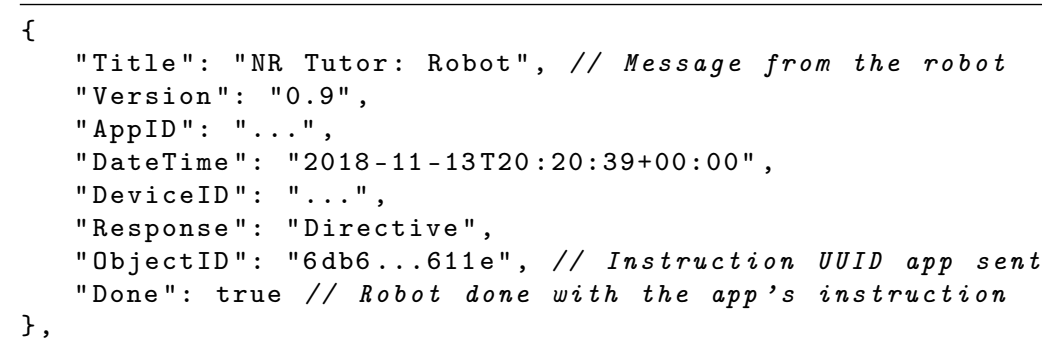

when adding additional software. Our version of NAO and Choregraphe used Python 2.7 as interpreter. Although this version of Python was supported when the NAO was originally released, it is no longer supported [36] as of today. This raised issues when we wanted to make the solution more secure and robust, as follows.

One specific issue arose while trying to implement the Transport Layer Security (TLS) for the solution. Since the the app and robot communicate together, it is good practice to run the connection over TLS to ensure the robot is talking to the correct app and the is talking to the correct robot. Unfortunately, this proved difficult to add certificates to the robot's certificate store and the required encryption algorithms were missing. As a result it was not possible to complete a TLS handshake between the robot and the app. Ultimately, the attempt was abandoned due to a lack of time. We instead focused on improving security by running the robot on a closed network.

Another issue came with the older version of the tornado library, where its websocket implementation did not handle the case of being unexpectedly disconnected correctly. There were features implemented in later versions of the library, but we could not upgrade as it was used by other parts of the system. It might have been possible to build a custom version of Python with the correct libraries, but this would have added additional complexity in how to communicate between the different Python processes. Again, we had to abandon this due to time shortage and came up with the send-and-confirmation protocol to add additional robustness to the solution.

\section{Trials}

There were two trials of the digital Language Shower. In both trials, the app was supposed to be tested also by a group of parents. Unfortunately, this proved difficult to achieve due to circumstances related to the Covid-19 pandemic. It was, thus, decided to drop the trials with parents and focus on the day-care centers instead. All trials were registered and approved by the Norwegian Centre for Research Data in advance.

\subsection{Pilot with beta prototype}

The beta versions of robot and app were tested in a pilot trial which was limited to 6-8 children in a single day-care center over a period of six days early in 2020. Only the words from a single Language Shower topic were introduced. The recruitment of a small population of children with a migration background was solely organized by the day-care center. The beta version was a proof of concepts and, thus, subject to many potential improvements.

At the beginning of the pilot, we had to solve several connectivity challenges, as the WebSocket connection to and from the robot was blocked by network firewalls at the day-care center. The situation was further complicated by the fact that the tablets in use were configured to connect to multiple networks (each with their own firewall) and would automatically choose the network with the strongest signal. As a consequence, the robot and tablets could suddenly be on different networks when the signal conditions changed, with the connection between the robot and tablet suddenly blocked via firewall. The remedy here was to restrict the robot and tablets to a single network. 
As outlined in Section 2.5, a Language Shower session lasted approximately $15 \mathrm{~min}$ utes, and its progress was controlled by the pedagogic staff. Researchers observed the session and how the children, staff, app, and robot interacted. They took notes, pictures, videos and interviewed the staff after the children had left. The observation and interview notes were analyzed, structured and turned into requirements for further improvement.

For instance, the staff were confused about how the robot was to be used, and this led to the app's how-to section being extended and partly re-written. Another example is that the day-care centers used iPads running iOS, and iOS does not support playback of audio files in the Ogg Vorbis format in a PWA. The solution was to replace all audio files with a format that are handled by both recent versions iOS and Android. It was also observed that the robot often classified answers as wrong, even though they were correct. The remedy here was to incorporate many more possible answers into the list of correct answers, and to loosen the checks for correct answers a bit.

The staff, in general, had a critical view towards Language Shower, which at that point was not conducted on a regular basis. In spite of the critical view, they commented that the program appeared to work well, and that the robot seemed to be "an exciting element of fun" in the language learning process. The staff particularly liked how the robot could be controlled by the app.

At the same time, the staff was concerned about how to ensure that answers were marked correctly. The robot would play an audio signal to indicate it was listening for the answer, but it was common that the children (often many at the same time) would answer the question before this signal was played. This lead to many situations were the ASR was not able to detect what had been answered, and, consequently, the robot stated the answer was wrong. Another topic was group size. The staff was concerned that a single adult would not be able to control the robot and simultaneously steer eight children aged three to six.

\subsubsection{Beta pilot summary and improvements}

The pilot showed that the digital version of the Language Shower seemed to fit in to the language learning work of the day-care centers. The quiz tasks and gamification elements in the app also appeared to increase the children's engagement. For instance, both staff and children appeared to be thrilled to have "a robot to play with", as one of the staff put it.

The pilot had also revealed several areas of improvement in particular on the technical side. First, it was critical to get the technology and the components up and running, and to communicate with each other. Speech recognition struggled when children spoke at the same time, and when the one answering was too far away to be heard. Even at the robot's microphones' highest sensitivity, the robot would sometimes not react at all. To our knowledge, solving this issue was beyond making adjustments to the robot. Therefore, the strategy was to instruct the staff that only a single child would answer a given question, and that each child needed to orient themselves so that they faced the front of robot's head. We also added functionality for multiple or repeated (typically three) answers, which could be configured in the content file. Overall, the observations and interviews lead to 81 major and minor improvements in the functionality, content, user interaction, and user interface.

\subsection{Main trial}

The main trial was conducted with the improved Language Shower over a period of six weeks in two day-care centers in the Grorud district in Autumn and Winter 2020, covering words from the three Language Shower topics presented in Section 2.5. As with the pilot, the recruitment of children was solely organized by the day-care center. In order for the participating testing groups to be sufficiently large, we had to accept a high degree of heterogeneity in age (3-5 years), language skills, as well as cultural and other background. A few days before the actual trial, the staff received a demonstration of how to handle the app and robot, and they were given a detailed instruction set and schedule for the lectures. 


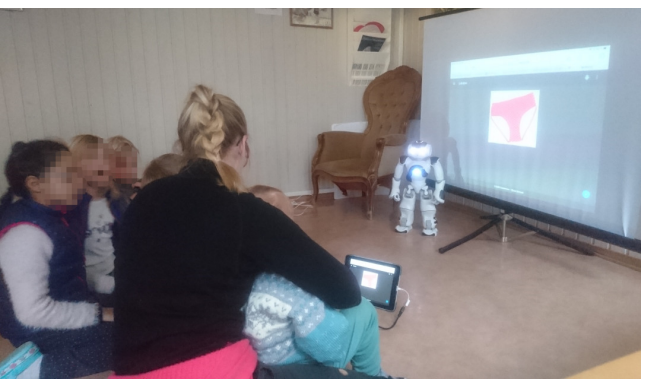

(a) Pilot

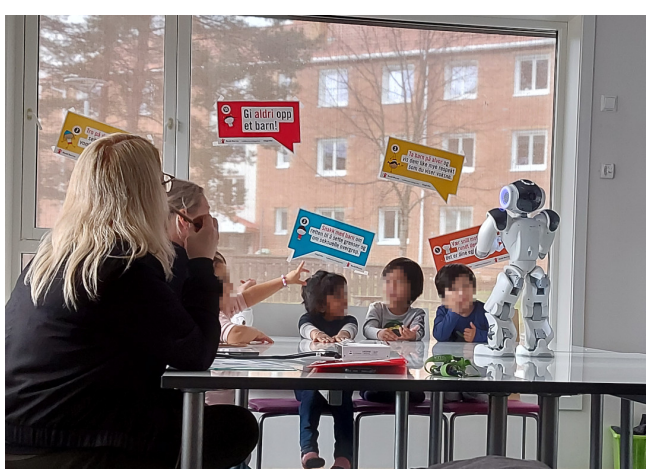

(b) Main trial

Figure 2. In situ stills from the trials

None of the children had been exposed to Language Shower earlier. Some of the staff had some experience with this program.

To measure the robot's impact, we had planned a quasi-experimental design with one robot group (RG) and one control group (CG) per day-care center. There were four groups in total: two RG and two CG. The robot groups should use both app and robot, while the control group would go through Language Shower in its original way. We had planned for seven children per group, 28 children in total, but this number was eventually reduced to 20 due to illness, "no show's", and other circumstances. For instance, a few children were simply too shy to participate on an equal level as compared to others.

All children had an immigrant background. The distribution of children per group is shown in Table 1. Both genders (10 female, 10 male) were sufficiently represented.

\subsubsection{Vocabulary tests}

The children's progress in language skills was measured in a pretest and a posttest. Each test counted the number of words that a child was able to recognize and say aloud, once before the trial, and once after. Each participating child was shown 33 small cards in total, one at a time, with pictures of words from Language Shower. The entire session was carried out in an informal and playful manner so that the children did not experience the session as a test. The pretest was conducted one week before the trial, whereas the posttest, which originally was planned for the week after the trial, had to be postponed by a few weeks due to the Covid-19 pandemic. The final time between the tests was 10 weeks. The delay affected the groups equally and therefore did not have an effect on the comparison across groups. One boy showed great progress during the trial and took the pretest in English and the posttest in Norwegian; this was considered as valid data.

Table 1: Distribution of gender over day-care centers and trial groups

\begin{tabular}{lrrrrr}
\hline & Girls & Boys & Sum & RG & CG \\
\hline Day-care center A & 6 & 6 & 12 & 6 & 6 \\
Day-care center B & 4 & 4 & 8 & 2 & 6 \\
\hline Sum & 10 & 10 & 20 & 8 & 12 \\
\hline
\end{tabular}

The number of correct words was counted for each child in each test, and the difference between pre- and posttest was then calculated, leading to a child's score. The average scores per trial group are shown in Table 2 along with this score as a percentage of the 33 total words in the program. Six children (3 out of 10) performed almost equally in the pre- and the posttest, with their scores being approximately zero $( \pm 1)$, i.e., they did not have any progression. For the majority, however, a substantial learning effect could be observed: On the average, each child in the robot group had learned 7.2 more words over 
the trial period, an improvement of $21.8 \%$. In the control group, the learning effect was a bit smaller: Here, a child had learned on the average 3.1 more words, an improvement of $9.4 \%$. This makes the robot group's progress approximately $12.4 \%$ larger than the control group's progress.

Table 2: Vocabulary scores per trial group.

\begin{tabular}{lcccc}
\hline & Min score & Max score & Average score & As percent of 33 words \\
\hline Robot Group & -1 & 13 & 7.2 & 21.8 \\
Control Group & -1 & 9 & 3.1 & 9.4 \\
\hline
\end{tabular}

While all groups in both day-care centers showed improvement in the tests and the RG did better than the CG in the same day-care center, the amount of progress in each center was different (Table 3). Children in day-care center A learned on average more words than those in day-care center B, regardless of if they were in RG or CG. In center A, the children in the robot group learned on the average 4.1 more words than in center $\mathrm{B}$, a difference of approximately $12.1 \%$ relative to the total of 33 words that the children had to go through. The same effect applies to the control group: In center A, the children had an average score 3.9 "words" higher than in center B, which had an average score of 1.3, a difference of around $11.9 \%$.

Table 3: Breakdown of vocabulary scores for day-care centers by group.

\begin{tabular}{lcc}
\hline & Average score & As percent of 33 words \\
\hline Robot Group A & 8.1 & 24.5 \\
Control Group A & 5.2 & 15.8 \\
\hline Robot Group B & 4 & 12.1 \\
Control Group B & 1.3 & 3.9 \\
\hline
\end{tabular}

\subsubsection{Observations}

We wanted to get a first-hand impression of the new Language Shower, to become familiar with the children's learning experience and the staff's user experience, and to note down any technical difficulties (and solve critical ones). So, we observed the children in-situ.

We had 13 observation sessions with all RGs and CGs in both participating day-care centers. Most of the observations were performed during the first and the last week of the trial. We expected excitement during the first week plus some potential technically critical challenges that needed to be solved. By waiting as long as possible between the first and final observation, we anticipated we would capture the biggest difference in the staff and children's interaction pattern. Due to the circumstances with the Covid-19 pandemic, three observations were conducted remotely, i.e., by video conferencing. This reduced the quality of the observation somewhat. The observations were noted down in writing and further documented with pictures and videos. These notes from the observations were then structured and analyzed such that we could see recurring topics and threads.

Table 4 provides a summary of the different observations. We can divide the observations into three categories: Technical issues, observations about the set up, and observation about the new language shower in general.

\subsubsection{Interviews with pedagogic staff}

We performed interviews with four of the pedagogic staff who were in charge of Language Shower for both RG and CG the participating day-care centers. The notes from the interviews were structured and analyzed.

A couple of comments considered the Language Shower program itself, i.e., without a robot. Staff from both day-care centers commented that a single adult running the sessions 
Table 4: Summary of observation from the observation sessions

\begin{tabular}{ll}
\hline Category & Observation \\
\hline & - The robot was very attractive for the children in the beginning \\
("Robbie is cool"), but the excitement diminished as technical & problems prevail ("this one's strange", "stupid robot"). \\
- In rare situations, app and robot were not in sync. \\
- When there was no reaction from the robot after being talked to, \\
it was unclear if it waited for an answer or not. \\
- The speech recognition should become more robust. The staff \\
should be able to override the speech recognition result in case. \\
- The speech recognition should be more robust regarding \\
wrongly pronounced words. For instance, many children replace \\
the difficult-to-pronounce Norwegian 'r' with an '1', so they said \\
"plikker" instead of "prikker" (in English: dots). \\
- The tablets' screens should always be connected to a projector, \\
such that pictures and tasks are easier to see. \\
- There should be as few distractions around the group as possi- \\
ble. \\
• It should be mandatory for the pedagogic staff to learn how the \\
robot can be used. \\
- The group in general needed more time to discuss after the robot \\
has asked a question. \\
• The learning scores and sound effects are liked by both children \\
and staff. \\
• All robot dances and songs were very well received. \\
• Dance moves and gestures should in general vary a lot. \\
General
\end{tabular}


was, in most cases, not sufficient, particularly with children requiring a lot of attention, and in case the technology would fail. While the number of children per group varied between five and eight during the trials, several staff members considered six to be the maximum group size.

There were multiple opinions regarding the children's age. The children that participated were aged three to six. One staff member believed the tasks to be too difficult for the youngest children. Another thought the words were too easy for the oldest children. A third informant thought it would be better to deploy Language Shower early in school.

Several felt that the groups had too heterogeneous in the trials, and more homogeneous groups would be beneficial for the success of Language Shower. Some children had dropped out in both RG and CG. The staff's explanation for this was that the most skilled children had quickly lost interest, while a few children were afraid of answering incorrectly.

Obviously, there were different preferences regarding language learning programs among the staff. One staff member recommended copying elements from other programs, for instance the program Sproff [37] that had been used earlier in day-care centers. Other staff had experienced that the children "more quickly become uneasy" with Sproff, and that the "learning factor with Language Shower was higher". Yet another informant expressed their dislike of Language Shower and said that it had little impact and was not directly related to the center's other language teaching efforts. In particular, the repetition part was viewed as being "a bit boring", and mind maps were considered to be unsuited for the youngest. This contradicted the opinion of another staff member who pointed out that the possibility to go into depth with every single picture in Language Shower, thus making each task a mind map, is one of the most important properties of the program. A clearly positive impact of Language Shower was mentioned: a girl was observed to talk exclusively during the sessions, but would otherwise remain mute. One staff member expressed indifference towards language learning programs and said "surely there will come something new soon". Approximately half of the staff had intentions to continue with Language Shower (with or without the robot).

There were mixed but mostly negative reactions for Language Shower with a robot. On the positive side, the sessions had been "very exciting" and "fun to have been a part of". The majority of the staff were positively surprised, as they had expected more chaos and distractions. This can be interpreted as a sign of the children's larger engagement and excitement when a robot is present. However, it was pointed out that this might vary a lot; for children with behavioral difficulties, a robot was not expected to solve all attention and motivation issues. Songs and dances were according to the pedagogic staff the most popular part of the Language Shower with the robot. One child was even so enthusiastic about the dances that she wished for a Nao robot as a Christmas present.

Several technical difficulties were mentioned, and many felt the trial had been a "varied experience". For instance, the technology had not always worked as expected, the robot would sometimes "hang up" or "freeze", it would not react when talked to. Occasionally it would reject answers as wrong with only slight variations in the pronunciation, and with perfectly valid speech by native speakers. This had, not surprisingly, had a negative effect on those who were still in the early process of learning the correct pronunciation. We were also told that the gap between what was said and what the robot understood in a few circumstances could be substantial; for instance, "hand" became "kebab", or "big toe" became "fotball".

In some situations, units had been misunderstood by the staff. Sometimes a staff member thought the robot was waiting for an answer, while in fact it was not. Or, the robot assumed that an answer had been given and went on to the next task, while, in fact, the group was still discussing, or a child was just talking quite slowly. This could lead to confusion later on regarding whether the robot was showing the correct picture or would show other "strange behavior". According to the staff, recognizing speech correctly required that the children were sitting very close in front of the robot, which could be challenging when a larger group of children were sitting around it. All these problems had 
lead to a negative impression for the staff, and one informant stated that the robot would "not be missed by anybody in the future".

The pedagogical staff's experiences have been turned into recommendations, which are summarized in the following. While many of these regard Language Shower in general, others target the robot, for instance "more dances".

- The group size should be 4-6 children.

- Groups should be more homogeneous with regard to skill level.

- An age of four was considered optimal.

- Consider differentiating with multiple levels of difficulty (as an alternative to more homogeneous groups and an optimal age).

- More effort should be put into the proper choice of appealing pictures and songs.

- There should be more dances [for the robot].

- There should be a lot of variation in task type, robot moves, sounds, standard answers, etc.

- The repetition of words should be less monotonous.

- Consider employing learning elements from different language programs, such as the aforementioned "Sproff".

\subsubsection{Main trial summary}

The trial shows a diversity of results. On the positive side, the quantitative results from the vocabulary tests tell us that there is a noticeable positive impact of using a social robot in the language learning process. As observers we witnessed a lot of engagement and excitement. On the negative side, it appears that the robot cannot compensate for limitations of the underlying language learning program. Technical difficulties further appeared to reduce the learning outcome and the user experience for the children and staff. Unfortunately, the problems with the technology were considered as so severe that the district authorities decided, among other reasons, to discontinue the Language Shower with a robot. The interviews supplemented the same conclusions as we had found in our observations.

It appears that the pedagogic staff are a key factor for the success of the language sessions. As long as they are not convinced by the program's potential for the children's language development, it is of little use that the program comes nicely wrapped in form of an attractive app and a cute, cool robot. Another important factor is the staff's ability to engage as teachers, with or without robot. For instance, we witnessed a pedagogue who was able to motivate and include all children equally as she carried out Language Shower without the robot; all children in the group seemed to have a great learning experience.

\section{Discussion}

The trials have uncovered benefits and disadvantages of the digital Language Shower program. Some of the critique points to technical matters, whereas other shortcomings can be linked to the original language learning program. Yet other issues are related to the staff and their acceptance of Language Shower per se.

Most importantly, the robot resulted in $13 \%$ faster language learning, a consistent effect seen in both day-care centers. The progress, however, was unevenly distributed. Some children showed no progress at all, or even a negative progress. The latter can be attributed to natural fluctuation of the experiment, while the former can have many complex explanations, such as differences in age, maturity, cultural background, family constellations, in how much the children's parents support language learning at home, as well as other, including combinations thereof. Unfortunately, the qualitative nature of the experiment did not provide the opportunity to control these parameters.

Our observations and measurements confirm the embodiment effect [38] social robots have for their surroundings. The effect can be observed with both children and adults as the combination of technology, semi-autonomous behavior, and human, child-like shape, and it manifests itself as fascination and increased attention and engagement, which in turn leads to improved learning. Music and dance were not used so much for learning in 
the Language Shower, but more for entertainment and engagement. Yet it might be this mix of entertainment and education that made is so appealing to both young and old. The effect's objective influence on the learning progress is reflected by the numbers from the vocabulary tests; however, as observations and interviews revealed, the fascination may diminish because of the continual technical difficulties. Paraphrased, there are limits to how engaging a robot can be, and future evaluations of the embodiment factor should therefore incorporate measurements of the user experience to reflect overall satisfaction with the technology, and how the robot is perceived and accepted. There is some evidence for the limits of embodiment, as the most skilled children soon lost interest or even dropped out of the robot group.

There are a few other aspects related to embodiment: With its height of about $58 \mathrm{~cm}$ and a "cute" look, none of the children we observed perceived NAO as a threat. However, we know from related work with autistic children that a humanoid robot of that size could trigger serious reactions of fright [39]. Drawing a parallel to other phobias of, say, mice, it might not be the robot's dimensions that trigger this condition. In our opinion it matters that the robot is small and comparable to the dimensions of an animal. As such, it can also can be placed on a table, and the children sit around it, as depicted in Figure 2(b). Moreover, for both Oslo municipality and the staff in the day-care centers, it was important that the robot appeared genderless (i.e., the robot presented neither as a boy nor a girl). It was therefor an advantage that the NAO 6 was available in gray only, i.e., without potentially "gendered colors", such as blue or pink. In addition, the staff chose the name "Robbie" for the robot since they felt that this name could apply equally well for a boy or girl.

As we had observed in the trials, the embodiment factor can, to a certain extent, make up for or draw attention away from weaknesses in the pedagogical concept, particularly in the beginning of a course. But there are limits to how much it can cover these weaknesses. For instance, if a mind map is not suitable for the youngest children, then neither a robot giving the task nor one helping with complementing the mind map will solve the situation. Such weaknesses will persist, and a robot might even contribute to the imperfections with its own peculiarities. A suitable example for the latter is that the majority of children quickly becomes bored when there was too little variation in the robot's responses or music and dances, which also illustrates another boundary of the embodiment factor.

Pre-school children, i.e., those aged three to six, have vary widely in their maturity and capabilities, including language learning. During the main trial, there were up to eight children with potentially wide-ranging abilities per group. This complicates finding an adequete level of difficulty in the learning material. We argue that personalized learning for each child in the group by means of a robot might be intricate to achieve, as the proper handling of several individuals at the same time requires sophisticated and flexible programming of the robot's logic. It might, therefore, be the best option with today's state of technology to deploy the robot in a single one-to-one setting. An alternative could be to offer series of tasks (within a single lecture) with increasing difficulty, such that the learning effect is highest in the beginning for the youngest and highest in the end for the oldest. Yet another possibility is to offer multiple lectures with multiple difficulties for multiple ages and allow for heterogeneous groups.

Given the issues we highlighted in Section 1.2 and our context of language learning with a small group of children and a teacher or pedagogue, it appears the technology is (still) insufficient to handle all issues. That is, a robot requires the proper detection and potentially identification of multiple speakers, as well as the handling of simultaneous speech, all of which are not easily solved as of today. Further, language learning, which often relies on small differences in pronunciation, demands for robust speech recognition with support for imperfect pronunciation, language accents, and varied dialects, as well as recognizing new or made-up words. To our knowledge, there are no good solutions for these challenges with today's technology. Solving these issues is particularly important in the context of small (and insecure) children. As illustrated in our trials, some of the 
insecure children dropped out of the robot group due to their fear of answering "wrong", even though the answer was actually correct but not understood by the robot.

Finally, latency in transmission and processing of data might add to the confusion of children and adults, as they then may be unable to determine whether or not the robot is waiting for an answer, or that the robot has received the spoken text and is instead waiting for a response from the cloud-based ASR service. The robot is supposed to make a sound when it is "listening", but sometimes this sound was delayed, not played at all, or even at wrong times. We suspect that problem was related to a bug in the robot's software. Regardless, this flaw contributed to further decrease children's confidence.

\section{Limitations}

The findings in this study are limited by the following considerations.

The trials have been bound to the tight project budget, with a total of three day-care centers, 34 participating children, valid vocabulary test data from 20 children and interviews with seven staff. It could easily be argued that these numbers are not representative for immigrant children in Norway. In a research project like this, however, it is virtually impossible to control the population of children and parents to be representative in an experiment design like ours, while keeping all other factors of comparison equal. Still, these numbers are in the majority of cases higher then those mentioned in other scientific literature.

The findings are affected by multiple and subtle factors. For instance, different rooms and surroundings, time of the day for the session, influence of the observers, as well as different interpretations of answers may have impacted each individual result of the vocabulary test. We cannot exclude the possibility that a particular child during the second test has become more confident when interacting with the researcher, leading to a positive impact on the result. It may further be assumed that each child would develop language skills naturally as time passes by without having been exposed to Language Shower. This factor would, however, have affected both the robot group and the control group in the same manner.

Finally, it is a weakness of the study that the effect of the developed solution on the children's parents could not have been sufficiently studied. Given the situation with the Covid-19 pandemic, we were satisfied to complete the trials with meaningful results. It has been difficult and time-consuming to come in contact with and consistently involve this important target group in the trials over time.

\section{Conclusion}

We have described how social robots can be used to strengthen children's training of a foreign language in day-care centers. The Language Shower was transformed into a mobile app with the option for additional enhancement a humanoid robot to lead lectures. The solution was tested in two major trials over the duration of multiple weeks in several day-care centers in the Grorud district in Oslo municipality in Norway.

Our measurements indicate a substantial positive impact by using the social robot as compared to the same course without robot. Our observations in situ and interviews with day-care center staff have at the same time disclosed a number of challenges with the educational concept as well as the underlying technology. Ultimately, the combination of these issues led to the district not continuing the use of Language Shower with the robot.

Future efforts of working incorporating a robot with language learning must consider ASR. ASR needs to improve and be made more flexible and robust. Other areas to explore should be recognizing and handling simultaneous answers, which is linked to multiplespeaker detection.

Our study met with several diverse, intricate issues spanning from obstacles imposed by the Covid-19 pandemic to technical issues with the robot and ASR. Issues with imperfect or failing technology play an important role for adopting new technology, and this (along with other factors) led to discontinuation in our case. For using social robots, our study 
showed us that synchronization issues and problems with ASR must be improved before performing new trials with a similar set up in other settings. To address other intricate issues, such as attitudes from the staff, behavior of the children, and the impact of the Covid-19 pandemic, the research design must take into account such obstacles, so that the negative effects can be mitigated as much as possible. Beyond technology, good cooperation with the staff of the day-care centers and the district leadership is important to ensure a project's success. Despite the obstacles we encountered during the study, we still are convinced that there is a role for social robots in language learning in day-care centers, and that this should be investigated further. The positive learning outcome measured from our sample supports this view.

Author Contributions: The authors have contributed to this article as follows. Conceptualization: Solheim, Halbach; methodology: Solheim; software: Halbach, Schulz; validation: Leister, Halbach; investigation: Halbach, Schulz; data curation: Halbach; writing-original draft preparation: Halbach; writing-review and editing: Leister, Schulz, Halbach; supervision: Leister; project administration: Solheim, Halbach; funding acquisition: Solheim

Funding: This research has been supported through funding by the Delprogram oppvekst og utdanning of Oslo municipality, district Grorud.

Institutional Review Board Statement: The study was conducted according to the guidelines of the Declaration of Helsinki, and approved by the the Norwegian Centre for Research Data (NSD) in advance, reference number 331729, dated 29 July, 2019.

Informed Consent Statement: Informed consent was obtained from all subjects involved in the study, respectively their parents.

Acknowledgments: We thank the pedagogues from Oslo municipality's district Grorud for advice regarding Language Shower, Margareth Sandvik at OsloMet for advice with the vocabulary tests, the day-care center staff for their participation and comments, and in particular the administrative staff in the district for help with setting up and carrying out the trials. Our project partner Innocom AS delivered the NAO 6 robots used in the project and contributed with technical expertise. We are also grateful to our summer students for helping with content production and vocabulary tests. Last but not least, we thank all parents that let their children participate in this study.

Conflicts of Interest: The authors declare no conflict of interest.The funding organization had no role in the design of the study; in the collection, analyses, or interpretation of data; in the writing of the manuscript, or in the decision to publish the results.

\section{Abbreviations}

The following abbreviations are used in this manuscript:

$\begin{array}{ll}\text { API } & \text { Application Programming Interface } \\ \text { ASR } & \text { Automatic Speech Recognition } \\ \text { CG } & \text { Control Group } \\ \text { HRI } & \text { Human-Robot Interaction } \\ \text { ICT } & \text { Information and Communication Technologies } \\ \text { IP } & \text { Internet Protocol } \\ \text { JSON } & \text { JavaScript Object Notation } \\ \text { LRS } & \text { Learning Record Store } \\ \text { PWA } & \text { Progressive Web Application } \\ \text { RALL } & \text { Robot-Assisted Language Learning } \\ \text { RG } & \text { Robot Group } \\ \text { TLS } & \text { Transport Layer Security } \\ \text { TTS } & \text { text to speech }\end{array}$

\section{References}

1. Fantoft, S. Hvert ord teller. In Områdesatsingene i Oslo: Årsmelding 2019; Oslo Kommune Byrådsavdeling for byutvikling, 2019; pp. 56-59. 
2. Breazeal, C.; Dautenhahn, K.; Kanda, T. Social Robotics. In Springer Handbook of Robotics; Siciliano, B.; Khatib, O., Eds.; Springer International Publishing, 2016; pp. 1935-1972. doi:10.1007/978-3-319-32552-1_72.

3. Fuglerud, K.S.; Solheim, I. The Use of Social Robots for Supporting Language Training of Children. Studies in Health Technology and Informatics. IOS Press, 2018, Vol. 256, pp. 401-408. doi:10.3233/978-1-61499-923-2-401.

4. Gouaillier, D.; Hugel, V.; Blazevic, P.; Kilner, C.; Monceaux, J.; Lafourcade, P.; Marnier, B.; Serre, J.; Maisonnier, B. Mechatronic Design of NAO Humanoid. 2009 IEEE International Conference on Robotics and Automation, 2009, pp. 769-774. doi:10.1109/ROBOT.2009.5152516.

5. Schulz, T.; Halbach, T.; Solheim, I. Using Social Robots to Teach Language Skills to Immigrant Children in an Oslo City District. Companion of the 2020 ACM/IEEE International Conference on Human-Robot Interaction; Association for Computing Machinery: Cambridge, United Kingdom, 2020; HRI '20, pp. 442-444. doi:10.1145/3371382.3378257.

6. Bartneck, C.; Forlizzi, J. A Design-Centred Framework for Social Human-Robot Interaction. RO-MAN 2004. 13th IEEE International Workshop on Robot and Human Interactive Communication (IEEE Catalog No.04TH8759); IEEE: Kurashiki, Okayama, Japan, 2004; pp. 591-594. doi:10.1109/ROMAN.2004.1374827.

7. Cooney, M.; Leister, W. Using the Engagement Profile to Design an Engaging Robotic Teaching Assistant for Students. Robotics 2019, 8, 21. doi:10.3390/robotics8010021.

8. Alemi, M.; Meghdari, A.; Ghazisaedy, M. Employing Humanoid Robots for Teaching English Language in Iranian Junior High-Schools. International Journal of Humanoid Robotics 2014, 11, 1450022. doi:10.1142/S0219843614500224.

9. Fels, D.; Waalen, J.; Zhai, S.; Weiss, P. Telepresence under Exceptional Circumstances: Enriching the Connection to School for Sick Children. Proc. of IFIP INTERACT01: Human-Computer Interaction, 2001, pp. 617-624.

10. Yamazaki, R.; Nishio, S.; Ogawa, K.; Ishiguro, H.; Matsumura, K.; Koda, K.; Fujinami, T. How Does Telenoid Affect the Communication between Children in Classroom Setting? CHI '12 Extended Abstracts on Human Factors in Computing Systems; ACM: New York, NY, USA, 2012; CHI EA '12, pp. 351-366. doi:10.1145/2212776.2212814.

11. Børsting, J.; Culén, A.L. A Robot Avatar: Easier Access to Education and Reduction in Isolation? Proceedings of the International Conference on E-Health 2016. IADIS, 2016, pp. 34-44.

12. Shiomi, M.; Kanda, T.; Howley, I.; Hayashi, K.; Hagita, N. Can a Social Robot Stimulate Science Curiosity in Classrooms? International Journal of Social Robotics 2015, 7, 641-652. doi:10.1007/s12369-015-0303-1.

13. Köse, H.; Uluer, P.; Akalın, N.; Yorganc1, R.; Özkul, A.; Ince, G. The Effect of Embodiment in Sign Language Tutoring with Assistive Humanoid Robots. International Journal of Social Robotics 2015, 7, 537-548.

14. Kanda, T.; Hirano, T.; Eaton, D.; Ishiguro, H. Interactive Robots as Social Partners and Peer Tutors for Children: A Field Trial. Hum.-Comput. Interact. 2004, 19, 61-84. doi:10.1207/s15327051hci1901\\\&2_4.

15. Kanda, T.; Ishiguro, H. Communication Robots for Elementary Schools. Proceedings of AISB'05 Symposium Robot Companions: Hard Problems and Open Challenges in Robot-Human Interaction (Hatfield Hertfordshire), 2005, pp. 54-63.

16. Belpaeme, T.; Vogt, P.; van den Berghe, R.; Bergmann, K.; Göksun, T.; de Haas, M.; Kanero, J.; Kennedy, J.; Küntay, A.C.; Oudgenoeg-Paz, O.; Papadopoulos, F.; Schodde, T.; Verhagen, J.; Wallbridge, C.D.; Willemsen, B.; de Wit, J.; Geçkin, V.; Hoffmann, L.; Kopp, S.; Krahmer, E.; Mamus, E.; Montanier, J.M.; Oranç, C.; Pandey, A.K. Guidelines for Designing Social Robots as Second Language Tutors. International Journal of Social Robotics 2018, pp. 1-17. doi:10.1007/s12369-018-0467-6.

17. Kanero, J.; Geçkin, V.; Oranç, C.; Mamus, E.; Köntay, A.C.; Göksun, T. Social Robots for Early Language Learning: Current Evidence and Future Directions. Child Development Perspectives 2018. doi:10.1111/cdep.12277.

18. Schodde, T.; Hoffmann, L.; Stange, S.; Kopp, S. Adapt, Explain, Engage-A Study on How Social Robots Can Scaffold SecondLanguage Learning of Children. ACM Transactions on Human-Robot Interaction (THRI) 2019, 9, 6:1-6:27. doi:10.1145/3366422.

19. Riek, L.D. Wizard of Oz Studies in HRI: A Systematic Review and New Reporting Guidelines. Journal of Human-Robot Interaction 2012, 1, 119-136. doi:10.5898/JHRI.1.1.Riek.

20. Randall, N. A Survey of Robot-Assisted Language Learning (RALL). ACM Transactions on Human-Robot Interaction (THRI) 2019, 9, 7:1-7:36. doi:10.1145/3345506.

21. van den Berghe, R.; Verhagen, J.; Oudgenoeg-Paz, O.; van der Ven, S.; Leseman, P. Social Robots for Language Learning: A Review. Review of Educational Research 2019, 89, 259-295. doi:10.3102/0034654318821286.

22. Alves-Oliveira, P.; Sequeira, P.; Paiva, A. The Role That an Educational Robot Plays. 2016 25th IEEE International Symposium on Robot and Human Interactive Communication (RO-MAN). IEEE, 2016, pp. 817-822.

23. Zaga, C.; Lohse, M.; Truong, K.P.; Evers, V. The Effect of a Robot's Social Character on Children Task Engagement: Peer Versus Tutor. Proceedings of the 7th International Conference on Social Robotics, ICSR 2015; Tapus, A.; André, E.; Martin, J.C.; Ferland, F.; Ammi, M., Eds.; Springer: Germany, 2015; Lecture Notes in Computer Science, pp. 704-713. doi:10.1007/978-3-319-25554-5-0.

24. Chang, C.W.; Lee, J.H.; Chao, P.Y.; Wang, C.Y.; Chen, G.D. Exploring the Possibility of Using Humanoid Robots as Instructional Tools for Teaching a Second Language in Primary School. Educational Technology \& Society 2010, 13, $13-24$.

25. Goetz, J.; Kiesler, S.; Powers, A. Matching Robot Appearance and Behavior to Tasks to Improve Human-Robot Cooperation. The 12th IEEE International Workshop on Robot and Human Interactive Communication, 2003. Proceedings. ROMAN 2003. Ieee, 2003, pp. 55-60.

26. Kennedy, J.; Baxter, P.; Belpaeme, T. The Robot Who Tried Too Hard: Social Behaviour of a Robot Tutor Can Negatively Affect Child Learning. 2015 10th ACM/IEEE International Conference on Human-Robot Interaction (HRI). IEEE, 2015 , pp. 67-74. 
27. Baxter, P.; Ashurst, E.; Read, R.; Kennedy, J.; Belpaeme, T. Robot Education Peers in a Situated Primary School Study: Personalisation Promotes Child Learning. PLoS One 2017, 12, e0178126. doi:10.1371/journal.pone.0178126.

28. Kennedy, J.; Lemaignan, S.; Montassier, C.; Lavalade, P.; Irfan, B.; Papadopoulos, F.; Senft, E.; Belpaeme, T. Child Speech Recognition in Human-Robot Interaction: Evaluations and Recommendations. Proceedings of the 2017 ACM/IEEE International Conference on Human-Robot Interaction - HRI '17; ACM Press: Vienna, Austria, 2017; pp. 82-90. doi:10.1145/2909824.3020229.

29. Vogt, P.; van den Berghe, R.; de Haas, M.; Hoffman, L.; Kanero, J.; Mamus, E.; Montanier, J.; Oranç, C.; Oudgenoeg-Paz, O.; García, D.H.; Papadopoulos, F.; Schodde, T.; Verhagen, J.; Wallbridgell, C.D.; Willemsen, B.; de Wit, J.; Belpaeme, T.; Göksun, T.; Kopp, S.; Krahmer, E.; Küntay, A.C.; Leseman, P.; Pandey, A.K. Second Language Tutoring Using Social Robots: A Large-Scale Study. 2019 14th ACM/IEEE International Conference on Human-Robot Interaction (HRI), 2019, pp. 497-505. doi:10.1109/HRI.2019.8673077.

30. NN. Systematisk arbeid med ord og begreper: MÅL: «Å Forbedre Barnas Norsk-språklige Ferdigheter Til Skolestart» [Systematic work with words and terms: GOAL: To improve children's Norwegian language skills for the school start], 2018. in Norwegian.

31. The Advanced Distributed Learning Initiative. Experience API (xAPI) Standard. web pages, 2020. https://adlnet.gov/projects/ xapi/, accessed: 27 August, 2021.

32. Acapela Group. Text Tag Documentation: Acapela TTS For Mobile, 2016. http://doc.aldebaran.com/2-4/_downloads/audio_ system_acapelamobilitytexttags.pdf, accessed 27 August, 2021.

33. Apple, Inc.. Speech Synthesis Programming Guide, 2006.

34. Fette, I.; Melnikov, A. The WebSocket Protocol. RFC 6455, RFC Editor, 2011. http://www.rfc-editor.org/rfc/rfc6455.txt.

35. Pot, E.; Monceaux, J.; Gelin, R.; Maisonnier, B. Choregraphe: A Graphical Tool for Humanoid Robot Programming. ROMAN 2009 - The 18th IEEE International Symposium on Robot and Human Interactive Communication, 2009, pp. 46-51. doi:10.1109/ROMAN.2009.5326209.

36. Petersen, B. PEP 373 - Python 2.7 Release Schedule. https://legacy.python.org/dev/peps/pep-0373/, 2016.

37. Årsberetning 2020 [Annual Report 2020]. Utdanningsetaten, Oslo kommune [Dept. of education, Municipality of Oslo], 2020. https:/ / www.oslo.kommune.no/etater-foretak-og-ombud/utdanningsetaten/arsberetning-2020/?del=4-3, in Norwegian, accessed 27 August, 2021.

38. Deng, E.; Mutlu, B.; Mataric, M. Embodiment in socially interactive robots. arXiv preprint arXiv:1912.00312 2019.

39. Kumazaki, H.; Muramatsu, T.; Yoshikawa, Y.; Matsumoto, Y.; Ishiguro, H.; Kikuchi, M.; Sumiyoshi, T.; Mimura, M. Optimal Robot for Intervention for Individuals with Autism Spectrum Disorders. Psychiatry and Clinical Neurosciences 2020, 74, 581-586. doi:10.1111/pcn.13132. 